\title{
Isomorphy up to complementation
}

\author{
Maurice Pouzet and Hamza Si Kaddour \\ Dedicated with warmth and admiration to Adrian Bondy at the occasion \\ of his seventieth birthday
}

\begin{abstract}
Considering uniform hypergraphs, we prove that for every nonnegative integer $h$ there exist two non-negative integers $k$ and $t$ with $k \leq t$ such that two $h$-uniform hypergraphs $\mathcal{H}$ and $\mathcal{H}^{\prime}$ on the same set $V$ of vertices, with $|V| \geq t$, are equal up to complementation whenever $\mathcal{H}$ and $\mathcal{H}^{\prime}$ are $k$-hypomorphic up to complementation. Let $s(h)$ be the least integer $k$ such that the conclusion above holds and let $v(h)$ be the least $t$ corresponding to $k=s(h)$. We prove that $s(h)=h+2^{\left\lfloor\log _{2} h\right\rfloor}$. In the special case $h=2^{\ell}$ or $h=2^{\ell}+1$, we prove that $v(h) \leq s(h)+h$. The values $s(2)=4$ and $v(2)=6$ were obtained in [9].
\end{abstract}

AMS 2000 SUBJECT Classifications: Primary 05C50, 05C60.

KEYWORDS AND PHRASES: Graph, hypergraph, reconstruction, incidence matrices, Ramsey's theorem.

\section{Main results}

We extend to hypergraphs a reconstruction result about graphs obtained in $[9]$.

We start recalling the result. Let $V$ be a set of cardinality $v$ (possibly infinite). Two graphs $G$ and $G^{\prime}$ with vertex set $V$ are isomorphic up to complementation if $G^{\prime}$ is isomorphic to $G$ or to the complement $\bar{G}$ of $G$. Let $k$ be a non-negative integer, $G$ and $G^{\prime}$ are $k$-hypomorphic up to complementation if for every $k$-element subset $K$ of $V$, the induced subgraphs $G_{\uparrow K}$ and $G_{\uparrow K}^{\prime}$ are isomorphic up to complementation. A graph $G$ is $k$-reconstructible up to complementation if every graph $G^{\prime}$ which is $k$-hypomorphic to $G$ up to complementation is in fact isomorphic to $G$ up to complementation.

It is shown in [9] that two graphs $G$ and $G^{\prime}$ on the same set of $n$ vertices are equal up to complementation whenever they are $k$-hypomorphic up to complementation and $4 \leq k \leq n-4$ (see [10] for the case $k=n-3$ : it is shown that under the same hypothesis $G$ and $G^{\prime}$ are isomorphic up to complementation). It is also shown that 4 is the least integer $k$ such that

arXiv: $1501.05181 \mathrm{v} 1$ 
every graph $G$ having a large number $n$ of vertices is $k$-reconstructible up to complementation.

We show here that this result extends to uniform hypergraphs. Our result is the following (see the definitions in the next section).

Theorem 1. Let $h$ be a non-negative integer. There are two non-negative integers $k$ and $t, k \leq t$ such that two h-uniform hypergraphs $\mathcal{H}$ and $\mathcal{H}^{\prime}$ on the same set $V$ of vertices, $|V| \geq t$, are equal up to complementation whenever $\mathcal{H}$ and $\mathcal{H}^{\prime}$ are $k$-hypomorphic up to complementation.

Let $s(h)$ be the least integer $k$ such that there is some $t$ such that the the conclusion above holds and let $v(h)$ be the least $t$ corresponding to $k=s(h)$.

Theorem 2. $\quad$ 1. $s(h)=h+2^{\left\lfloor\log _{2} h\right\rfloor}$;

2. $v(h) \leq s(h)+h$ if $h=2^{\ell}$ or $h=2^{\ell}+1$.

Problem 1. Does $v(h)=s(h)+h$ for every $h$ ?

The proof of the result of [9] was based on a result of Wilson on the rank of incidence matrices over the two-element field [25]. Here, we use essentially Ramsey's theorem, Lucas's theorem and the notion of almost constant hypergraph. We use Ramsey's theorem and compactness theorem of first order logic in order to obtain the existence of $k$ and $t$ (see Theorem 8 and Claim 2). We use Lucas's theorem to prove (1) of Theorem 2 (we use it for the inequality $s(h) \geq h+2^{\left\lfloor\log _{2} h\right\rfloor}$ (see Theorem 10) and also for the reverse inequality (see Theorem 9), where we use also a decomposition result about hypergraphs (see Proposition 3)). With Wilson's theorem, we get both (1) and (2) when $h$ is of the form $2^{\ell}$ or $2^{\ell}+1$ (see Corollary 3 and Theorem 7).

In Section 5 of the paper we introduce a generalization of the notion of isomorphy up to complementation. We consider colorations of complete graphs and hypergraphs and isomorphy up to permutations of the colors. We raise some questions.

We refer to [5] for notions of graph theory. We use also basic notions of set theory, we denote by $\wp(V)$ the power set of $V$ and by $[V]^{h}$ the collection of $h$-element subsets of $V$.

\section{Hypergraphs, incidence matrices and almost constant hypergraphs}

\subsection{Isomorphy up to complementation}

Recall that a hypergraph is a pair $\mathcal{H}:=(V, \mathcal{E})$ where $\mathcal{E}$ is a collection of subsets of $V$; members of $V$ are the vertices of $\mathcal{H}$, whereas members of $\mathcal{E}$ 
are the hyperedges. We denote by $V(\mathcal{H})$, resp. $\mathcal{E}(\mathcal{H})$, the sets of vertices, resp. hyperedges, of a hypergraph $\mathcal{H}$; we denote by $v(\mathcal{H})$, resp. $e(\mathcal{H})$, the cardinality of $V(\mathcal{H})$, resp. $\mathcal{E}(\mathcal{H})$. If $E \in \mathcal{E}(\mathcal{H})$ we set $\mathcal{H}(E)=1$; otherwise we set $\mathcal{H}(E)=0$. If $K$ is a subset of $V$, the hypergraph induced by $\mathcal{H}$ on $V$ is $\mathcal{H}_{\uparrow K}=(K, \mathcal{E} \cap \wp(K))$. Let $h$ be an integer; the hypergraph $\mathcal{H}$ is $h$-uniform (or $h$-regular) if all its edges have size $h$ (for instance every graph is a 2uniform hypergraph); we make the convention that a hypergraph with no hyperedge is $h$-uniform for every $h$. With this convention, if $\mathcal{H}$ is $h$-uniform, the complement of $\mathcal{H}, \overline{\mathcal{H}}:=\left(V,[V]^{h} \backslash \mathcal{E}\right)$ is $h$-uniform.

Let $\mathcal{H}:=(V, \mathcal{E}), \mathcal{H}^{\prime}:=\left(V^{\prime}, \mathcal{E}^{\prime}\right)$, be two hypergraphs. An isomorphism from $\mathcal{H}$ onto $\mathcal{H}^{\prime}$ is any bijective map $f$ from $V$ onto $V^{\prime}$ such that the natural extension $\bar{f}$ to $\wp(V)$ induces a bijective map from $\mathcal{E}$ onto $\mathcal{E}^{\prime}$. If such a map exists, $\mathcal{H}$ and $\mathcal{H}^{\prime}$ are isomorphic. They are isomorphic up to complementation if either $\mathcal{H}$ is isomorphic to $\mathcal{H}^{\prime}$ or $\mathcal{H}$ is isomorphic to $\overline{\mathcal{H}^{\prime}}$. If $\mathcal{H}$ and $\mathcal{H}^{\prime}$ have the same set $V$ of vertices, we say that they are equal up to complementation if $\mathcal{H}=\mathcal{H}^{\prime}$ or $\mathcal{H}=\overline{\mathcal{H}^{\prime}}$; if moreover, the induced hypergraphs $\mathcal{H}_{\uparrow K}$ and $\mathcal{H}^{\prime}{ }_{\lceil K}$ are isomorphic up to complementation for all the $k$-element subsets of $V$, we say that $\mathcal{H}$ and $\mathcal{H}^{\prime}$ are $k$-hypomorphic up to complementation.

The relationship between $k$-hypomorphy for different values of $k$ is given by Proposition 1 below. The case of graphs is treated by Proposition 2.4 [9] by means of Gottlieb-Kantor theorem $[14,16]$. The general case follows the same lines.

\subsection{Incidence matrices}

Let $V$ be a finite set, with $v$ elements. Given non-negative integers $t, k$, let $W_{t k}$ be the $\left(\begin{array}{l}v \\ t\end{array}\right)$ by $\left(\begin{array}{l}v \\ k\end{array}\right)$ matrix of 0 's and 1 's, the rows of which are indexed by the $t$-element subsets $T$ of $V$, the columns are indexed by the $k$-element subsets $K$ of $V$, and where the entry $W_{t k}(T, K)$ is 1 if $T \subseteq K$ and is 0 otherwise.

A fundamental result, due to D.H. Gottlieb [14], and independently W. Kantor [16], is this:

Theorem 3. For $t \leq \min (k, v-k), W_{t k}$ has full row rank over the field $\mathbb{Q}$ of rational numbers.

If $k:=v-t$ then, up to a relabelling, $W_{t}$ is the adjacency matrix $A_{t, v}$ of the Kneser graph $K G(t, v)$, graph whose vertices are the $t$-element subsets of $V$, two subsets forming an edge if they are disjoint.

An equivalent form of Theorem 3 is:

Theorem 4. $A_{t, v}$ is non-singular for $t \leq \frac{v}{2}$. 
Applications to graphs and relational structures were given in [12] and [22]. Let us explain why the use of this result in our context is natural.

Let $X_{1}, \ldots, X_{r}$ be an enumeration of the $h$-element subsets of $V$; let $K_{1}, \ldots, K_{s}$ be an enumeration of the $k$-element subsets of $V$ and $W_{h k}$ be the matrix of the $h$-element subsets versus the $k$-element subsets. If $\mathcal{H}$ is a $h$ uniform hypergraph with vertex set $V$, let $w_{\mathcal{H}}$ be the row matrix $\left(g_{1}, \ldots, g_{r}\right)$ where $g_{i}=1$ if $X_{i}$ is a hyperedge of $\mathcal{H}, 0$ otherwise. We have $w_{\mathcal{H}} W_{h k}=$ $\left(e\left(\mathcal{H}_{\uparrow K_{1}}\right), \ldots, e\left(\mathcal{H}_{\uparrow K_{s}}\right)\right)$. Thus, if $\mathcal{H}$ and $\mathcal{H}^{\prime}$ are two hypergraphs with vertex set $V$ such that $\mathcal{H}_{\uparrow K}$ and $\mathcal{H}_{\lceil K}^{\prime}$ have the same number of hyperedges for every $k$-element subset of $V$, we have $\left(w_{\mathcal{H}}-w_{\mathcal{H}^{\prime}}\right) W_{h k}=0$. Thus, provided that $v \geq k+h$, by Theorem $3, w_{\mathcal{H}}-w_{\mathcal{H}^{\prime}}=0$ that is $\mathcal{H}=\mathcal{H}^{\prime}$.

Proposition 1. Let $v, k$ be non-negative integers, let $t \leq \min (k, v-k)$ and $\mathcal{H}$ and $\mathcal{H}^{\prime}$ be two h-uniform hypergraphs on the same set $V$ of $v$ vertices. If $\mathcal{H}$ and $\mathcal{H}^{\prime}$ are $k$-hypomorphic up to complementation then they are $t$ hypomorphic up to complementation.

Proof. Let $\mathcal{G}$ be a hypergraph on $t$ vertices. Set $I s(\mathcal{G}, \mathcal{H}):=\{L \subseteq V$ : $\left.\mathcal{H}_{\uparrow L} \simeq \mathcal{G}\right\}, I s c(\mathcal{H}, \mathcal{G}):=I s(\mathcal{G}, H) \cup I s(\overline{\mathcal{G}}, \mathcal{H})$ and $w_{\mathcal{G}, \mathcal{H}}$ the $0-1$-row vector indexed by the $t$-element subsets $X_{1}, \ldots, X_{r}$ of $V$ whose coefficient of $X_{i}$ is 1 if $X_{i} \in \operatorname{Isc}(\mathcal{G}, \mathcal{H})$ and 0 otherwise. From our hypothesis, it follows that $w_{\mathcal{G}, \mathcal{H}} W_{t k}=w_{\mathcal{G}, \mathcal{H}^{\prime}} W_{t k}$. From Theorem 3, this implies $w_{\mathcal{G}, \mathcal{H}}=w_{\mathcal{G}, \mathcal{H}^{\prime}}$ that is $\operatorname{Isc}(\mathcal{G}, \mathcal{H})=\operatorname{Isc}\left(\mathcal{G}, H^{\prime}\right)$. Since this equality holds for all hypergraphs $\mathcal{G}$ on $t$-vertices, the conclusion of the proposition follows.

In particular, two $h$-uniform hypergraphs $\mathcal{H}$ and $\mathcal{H}^{\prime}$ on the same set $V$ of vertices, $|V| \geq 2 k-1$, are $k^{\prime}$-hypomorphic up to complementation for every $k^{\prime} \leq k$ provided that there are $k$-hypomorphic up to complementation.

As noted by a referee of this paper, Proposition 1 can be obtained directly by a counting argument similar to Kelly's lemma (cf Lemma 2.20 p. 67 [5]). A generalization of Kelly's lemma for such a type of result was in [23] which appeared after [22].

A fundamental result, due to R. M. Wilson [25], is the following.

Theorem 5. (R. M. Wilson [25]) For $t \leq \min (k, v-k)$, the rank of $W_{t k}$ modulo a prime $p$ is

$$
\sum\left(\begin{array}{l}
v \\
i
\end{array}\right)-\left(\begin{array}{c}
v \\
i-1
\end{array}\right)
$$

where the sum is extended over those indices $i, 0 \leq i \leq t$, such that $p$ does not divide the binomial coefficient $\left(\begin{array}{c}k-i \\ t-i\end{array}\right)$.

In the statement of the theorem, $\left(\begin{array}{c}v \\ -1\end{array}\right)$ should be interpreted as zero. 
We will apply Wilson's theorem to $h$-uniform hypergraphs, for $p=2$, $t=h, k=2 r h$ where $r$ is a positive integer. In this case we will obtain that the rank of $W_{h k}(\bmod 2)$ is $\left(\begin{array}{l}v \\ h\end{array}\right)-1$.

Let $n, p$ be positive integers, the decomposition of $n=\sum_{i=0}^{n(p)} n_{i} p^{i}$ in the basis $p$ is also denoted $\left[n_{0}, n_{1}, \ldots, n_{n(p)}\right]_{p}$ where $n_{n(p)} \neq 0$ if and only if $n \neq 0$.

Theorem 6. (Lucas's Theorem [19]) Let $p$ be a prime number, $t, k$ be positive integers, $t \leq k, t=\left[t_{0}, t_{1}, \ldots, t_{t(p)}\right]_{p}$ and $k=\left[k_{0}, k_{1}, \ldots, k_{k(p)}\right]_{p}$. Then

$$
\left(\begin{array}{l}
k \\
t
\end{array}\right)=\prod_{i=0}^{t(p)}\left(\begin{array}{c}
k_{i} \\
t_{i}
\end{array}\right)(\bmod p), \text { where }\left(\begin{array}{c}
k_{i} \\
t_{i}
\end{array}\right)=0 \text { if } t_{i}>k_{i} .
$$

For an elementary proof of Theorem 6, see Fine [11]. We will use the following well-known consequences of Theorem 6 .

Corollary 1. 1. Let $p$ be a prime and $t, k$ be positive integers, $t \leq k$, let $t=\left[t_{0}, t_{1}, \ldots, t_{t(p)}\right]_{p}$ and $k=\left[k_{0}, k_{1}, \ldots, k_{k(p)}\right]_{p}$.

(a) Then $p \mid\left(\begin{array}{l}k \\ t\end{array}\right)$ if and only if there is $i \in\{0,1, \ldots, t(p)\}$ such that $t_{i}>k_{i}$.

(b) $\left(\begin{array}{c}k \\ t\end{array}\right)$ is odd if, and only if, for all integers $i \in\{0,1, \ldots, t(2)\}$, $t_{i}=1 \Rightarrow k_{i}=1$.

2. Let $v \geq 2$. Then $v$ is a power of 2 if, and only if, $\left(\begin{array}{l}v \\ k\end{array}\right)$ is even for all $k \in\{1, \ldots, v-1\}$.

Corollary 2. Let $h$ and $k$ be positive integers. Then the rank of $W_{h k}$ modulo 2 is $\left(\begin{array}{l}v \\ h\end{array}\right)-1$ if and only if $h$ is a power of 2 and $k=2 r h$ where $r$ is a positive integer.

Proof. First we prove the converse implication. We have $h=2^{\ell}$ for some integer $\ell$ and $r=\sum_{j=0}^{t} \varepsilon_{j} 2^{j}$ for some $t$ with $\varepsilon_{t}=1$, then $k=\sum_{j=0}^{t} \varepsilon_{j} 2^{\ell+j+1}$. Let $s$ be the first integer $j$ such that $\varepsilon_{j} \neq 0$. We have $2^{\ell+s+1}=2^{l}+\sum_{p=0}^{s} 2^{l+p}$. For $i \leq h,\left(\begin{array}{c}k-i \\ h-i\end{array}\right)=\left(\begin{array}{c}\left(2^{l}-i\right)+\sum_{p=0}^{s} 2^{l+p}+\sum_{j=s+1}^{t} \varepsilon_{j} 2^{\ell+j+1} \\ 2^{l}-i\end{array}\right)$. Applying Lucas's theorem, $\left(\begin{array}{l}k \\ h\end{array}\right)$ is even and $\left(\begin{array}{l}k-i \\ h-i\end{array}\right)$ is odd if $i \neq 0$. Now by Wilson's theorem, the rank of $W_{h k}$ modulo 2 is $\left(\begin{array}{l}v \\ h\end{array}\right)-1$.

Let us prove the direct implication. Note that the rank of $W_{h k}$ modulo 2 is $\left(\begin{array}{l}v \\ h\end{array}\right)-1$ if and only if $\left(\begin{array}{c}k-i \\ h-i\end{array}\right)$ is odd for all $i \in\{1, \ldots, h\}$ and $\left(\begin{array}{l}k \\ h\end{array}\right)$ is even, in particular $k>h$. We have $h\left(\begin{array}{l}k \\ h\end{array}\right)=k\left(\begin{array}{l}k-1 \\ h-1\end{array}\right)$. Since $\left(\begin{array}{l}k \\ h\end{array}\right)$ is even and $\left(\begin{array}{l}k-1 \\ h-1\end{array}\right)$ is odd then $k$ is even. Now $(h-1)\left(\begin{array}{l}k-1 \\ h-1\end{array}\right)=(k-1)\left(\begin{array}{l}k-2 \\ h-2\end{array}\right)$. Since $\left(\begin{array}{l}k-1 \\ h-1\end{array}\right)$ and $\left(\begin{array}{l}k-2 \\ h-2\end{array}\right)$ are odd then $h$ and $k$ have the same parity. So $h$ and $k$ are even. 
We have $h=\left[h_{0}, h_{1}, \ldots, h_{h(2)}\right]_{2}$ and $k=\left[k_{0}, k_{1}, \ldots, k_{k(2)}\right]_{2}$ with $h_{0}=$ $k_{0}=0$.

First we prove that for all $i<h_{h(2)}, h_{i}=k_{i}$. By contradiction, let $j$ be the first integer $i \geq 1$ such that $h_{i} \neq k_{i}$. If $h_{j}=0$ and $k_{j}=1$, then $\left(h-2^{j}\right)_{j}=1$ and $\left(k-2^{j}\right)_{j}=0$, this contradicts the fact that $\left(\begin{array}{c}k-2^{j} \\ h-2^{j}\end{array}\right)$ is odd. So $h_{j}=1$ and $k_{j}=0$. Since $\left(\begin{array}{l}k-2^{h(2)} \\ h-2^{h(2)}\end{array}\right)$ is odd then $k_{h(2)}=0$. Let $n$ be the first integer $m>h(2)$ such that $k_{m} \neq 0$. We have $2^{n}-2^{h(2)}=2^{h(2)}+2^{h(2)+1}+\ldots+2^{n-1}$. Then $\left(k-2^{h(2)}\right)_{h(2)}=1$ and $\left(h-2^{h(2)}\right)_{h(2)}=0$. That contradicts the fact that $\left(\begin{array}{l}k-2^{h(2)} \\ h-2^{h(2)}\end{array}\right)$ is odd. So we have proved that for all $i<h_{h(2)}, h_{i}=k_{i}$. Then, since $\left(\begin{array}{l}k \\ h\end{array}\right)$ is even, we have $k_{h(2)}=0$. Thus, for all $i<h_{h(2)}, h_{i}=k_{i}=0$ since $\left(\begin{array}{l}k-2^{i} \\ h-2^{i}\end{array}\right)$ is odd. That gives $h=2^{\ell}$ for some integer $\ell$ and $k=\sum_{i=\ell+1}^{t} k_{i} 2^{i}$ for some integer $t$. Then $k=2 r h$ where $r$ is a positive integer.

Proposition 2. Let $h$ be a power of $2, k=2 r h$ where $r$ is a positive integer, and $\mathcal{H}$ and $\mathcal{H}^{\prime}$ be two h-uniform hypergraphs on the same set $V$ of $v \geq k+h$ vertices. Then the following properties are equivalent:

(i) $e\left(\mathcal{H}_{\uparrow K}\right)$ and $e\left(\mathcal{H}_{\uparrow K}^{\prime}\right)$ have the same parity for all $k$-element subsets $K$ of $V$;

(ii) $\mathcal{H}^{\prime}=\mathcal{H}$ or $\mathcal{H}^{\prime}=\overline{\mathcal{H}}$.

Proof. The implication $(i i) \Rightarrow(i)$ is trivial. We prove $(i) \Rightarrow(i i)$.

We have $h=2^{l}$ for some integer $l$. Let $W_{h k}$ be the matrix defined page 3 and ${ }^{t} W_{h k}$ its transpose. Let $U:=\mathcal{H} \dot{+} \mathcal{H}^{\prime}$. From the fact that $e\left(\mathcal{H}_{\uparrow K}\right)$ and $e\left(\mathcal{H}_{\uparrow K}^{\prime}\right)$ have the same parity for all $k$-element subsets $K$, the boolean sum $U$ belongs to the kernel of ${ }^{t} W_{h k}$ over the 2-element field. By Corollary 2, the rank of $W_{h k}$ modulo 2 is $\left(\begin{array}{l}v \\ h\end{array}\right)-1$. Then the kernel of its transpose ${ }^{t} W_{h k}$ has dimension 1 . Since $(1, \cdots, 1) W_{h k}=(0, \cdots, 0)(\bmod 2)$ then $w_{U} W_{h k}=$ $(0, \cdots, 0)(\bmod 2)$ amounts to $w_{U}=(0, \cdots, 0)$ or $w_{U}=(1, \cdots, 1)$, that is $U$ is empty or complete, so $\mathcal{H}^{\prime}=\mathcal{H}$ or $\mathcal{H}^{\prime}=\overline{\mathcal{H}}$.

Corollary 3. Let $h$ be a power of $2, k=2 r h$ where $r$ is a positive integer, and $\mathcal{H}$ and $\mathcal{H}^{\prime}$ be two h-uniform hypergraphs on the same set $V$ of $v \geq k+h$ vertices. If $\mathcal{H}$ and $\mathcal{H}^{\prime}$ are $k$-hypomorphic up to complementation then $\mathcal{H}^{\prime}=\mathcal{H}$ or $\mathcal{H}^{\prime}=\overline{\mathcal{H}}$.

Proof. From Corollary 1, $\left(\begin{array}{l}k \\ h\end{array}\right)$ is even. Then we conclude using Proposition 2.

Theorem 7. Two $\left(2^{\ell}+1\right)$-uniform hypergraphs $\mathcal{H}$ and $\mathcal{H}^{\prime}$ on the same set $V$ of $v$ vertices, $v \geq 3.2^{\ell}+2$ and $\ell \geq 1$, are equal up to complementation whenever $\mathcal{H}_{\uparrow K}$ and $\mathcal{H}_{\uparrow K}^{\prime}$ have the same number of edges up to complementation for all $k$-element subsets $K$ of $V$ for $k \in\left\{2^{\ell+1}, 2^{\ell+1}+1\right\}$. 
Proof. Let $U:=\mathcal{H} \dot{+} \mathcal{H}^{\prime}$. By Corollary $1,\left(\begin{array}{c}2^{\ell+1} \\ 2^{\ell}+1\end{array}\right)$ and $\left(\begin{array}{c}2^{\ell+1}+1 \\ 2^{\ell}+1\end{array}\right)$ are even. Then for $k \in\left\{2^{\ell+1}, 2^{\ell+1}+1\right\}, e\left(\mathcal{H}_{\uparrow K}\right)$ and $e\left(\mathcal{H}_{\uparrow K}^{\prime}\right)$ have the same parity for all $k$ element subsets $K$ of $V$. Hence $w_{U}$ belongs to the kernel of ${ }^{t} W_{2^{\ell}+1 k}$ over the 2-element field, for $k \in\left\{2^{\ell+1}, 2^{\ell+1}+1\right\}$. By Corollary 1 and Theorem 5 , the rank of $W_{2^{\ell}+12^{\ell+1}}$ modulo 2 is $v-1+\left(\begin{array}{c}v \\ 2^{\ell}+1\end{array}\right)-\left(\begin{array}{c}v \\ 2^{\ell}\end{array}\right)$, the rank of $W_{2^{\ell}+1} 2^{\ell+1}+1$ modulo 2 is $\left(\begin{array}{c}v \\ 2^{\ell}+1\end{array}\right)-v$, the second rank is obvious to obtain; the first one uses this: $\left(\begin{array}{c}2^{\ell+1}-i \\ 2^{\ell}+1-i\end{array}\right)$ is odd for $i=1,2^{\ell}+1$; for $i$ even $\left(\begin{array}{c}2^{\ell+1}-i \\ 2^{\ell}+1-i\end{array}\right)$ is even; for $i$ odd, $3 \leq i \leq 2^{\ell}-1$, set $j:=i-1$, then $j$ is even and $2 \leq j \leq 2^{\ell}-2$, we have $\left(\begin{array}{c}2^{\ell+1}-i \\ 2^{\ell}+1-i\end{array}\right)=\left(\begin{array}{c}2^{\ell}+2^{\ell}-1-j \\ 2^{\ell}-j\end{array}\right), 2^{\ell}-j=\sum_{q=s}^{t} \theta_{q} 2^{q}$ with $\theta_{s}=\theta_{t}=1$, thus $2^{\ell}-1-j=\sum_{q=0}^{s-1} 2^{q}+\sum_{q=s+1}^{t} \theta_{q} 2^{q}$, that shows that $\left(\begin{array}{c}2^{\ell+1}-i \\ 2^{\ell}+1-i\end{array}\right)$ is even. Then the dimension of $\operatorname{Ker}\left({ }^{t} W_{2^{\ell}+12^{\ell+1}}\right)$ is $\left(\begin{array}{c}v \\ 2^{\ell}\end{array}\right)-v+1$ and the dimension of $\operatorname{Ker}\left({ }^{t} W_{2^{\ell}+1} 2^{\ell+1}+1\right)$ is $v$. Note that $(1,1, \ldots, 1) \in$ $\operatorname{Ker}\left({ }^{t} W_{2^{\ell}+12^{\ell+1}}\right) \cap \operatorname{Ker}\left({ }^{t} W_{2^{\ell}+1} 2^{\ell+1}+1\right)$.

Let $X_{1}, \ldots, X_{r}$ be the enumeration of the $\left(2^{\ell}+1\right)$-element subsets of $V$ which appears as rows of the matrices $W_{2^{\ell}+12^{\ell+1}}$ and $W_{2^{\ell}+12^{\ell+1}+1}$. For $a \in V$, we set $v_{a}:=\left(\varepsilon_{1}, \ldots, \varepsilon_{r}\right)$ where $\varepsilon_{i}=1$ if $a \in X_{i}, 0$ otherwise. We have ${ }^{t} v_{a} \in \operatorname{Ker}\left({ }^{t} W_{2^{\ell}+1} 2^{\ell+1}+1\right)$. Note that for all $A \subseteq V, A \neq \emptyset, \sum_{a \in A}{ }^{t} v_{a} \neq 0$, indeed if $\sum_{a \in A}{ }^{t} v_{a}=0$ then $A \neq V$ and $\left|A \cap X_{i}\right|$ is even for all $i \in\{1, \ldots, r\}$. Let $i_{0}$ be such that $A \cap X_{i_{0}} \neq \emptyset$. Let $u \in A \cap X_{i_{0}}$ and $w \in V \backslash A$, set $Y:=\left(X_{i_{0}} \backslash\{u\}\right) \cup\{w\}$. Then $Y$ is some $X_{i}$, but $|A \cap Y|$ is odd, that contradicts $\left|A \cap X_{i}\right|$ even for all $i$. So the family $\left\{{ }^{t} v_{a}: a \in V\right\}$ is linearly independent and thus forms a basis of $\operatorname{Ker}\left({ }^{t} W_{2^{\ell}+1} 2^{\ell+1}+1\right)$. Let $u \in \operatorname{Ker}\left({ }^{t} W_{2^{\ell}+1} 2^{\ell+1}\right) \cap$ $\operatorname{Ker}\left({ }^{t} W_{2^{\ell}+12^{\ell+1}+1}\right)$ with $u \neq 0$. Then $u=\sum_{a \in A}{ }^{t} v_{a}$ for some non-empty $A \subseteq V$. Since $u \in \operatorname{Ker}\left({ }^{t} W_{2^{\ell}+1} 2^{\ell+1}\right), \sum_{a \in A}{ }^{t} W_{2^{\ell}+1} 2^{\ell+1}{ }^{t} v_{a}=0$. It follows that $\sum_{a \in A \cap F}\left|\left\{X_{i}: a \in X_{i}, X_{i} \subseteq F\right\}\right|=0$ for every $2^{\ell+1}$-element subset $F$ of $V$ and thus $|A \cap F|$ is even. From that we deduce $A=V$. Indeed, if $A \neq V$, pick $b \in V \backslash A$, let $F_{1}$ be a $2^{\ell+1}$-element subset of $V$ such that $A \cap F_{1} \neq \emptyset$. Let $b_{1} \in A \cap F_{1}$, set $F:=\left(F_{1} \backslash\left\{b_{1}\right\}\right) \cup\{b\}$, then we have $|A \cap F|$ odd, a contradiction. Thus ${ }^{t} u=\sum_{a \in V} v_{a}=(1,1, \ldots, 1)$ proving that $\left\{{ }^{t}(1,1, \ldots, 1)\right\}$ forms a basis of $\operatorname{Ker}\left({ }^{t} W_{2^{\ell}+1} 2^{\ell+1}\right) \cap \operatorname{Ker}\left({ }^{t} W_{2^{\ell}+1} 2^{\ell+1}+1\right)$. Since ${ }^{t} w_{U} \in \operatorname{Ker}\left({ }^{t} W_{2^{\ell}+1} 2^{\ell+1}\right) \cap \operatorname{Ker}\left({ }^{t} W_{2^{\ell}+1} 2^{\ell+1}+1\right)$, then $w_{U}=(0, \cdots, 0)$ or $w_{U}=(1, \cdots, 1)$, that is $U$ is empty or complete, so $\mathcal{H}^{\prime}=\mathcal{H}$ or $\mathcal{H}^{\prime}=\overline{\mathcal{H}}$.

Problem 2. Extend the proof of Theorem 7 to others values of $h$. For an example, if $h=6, s(h)=10$. We guess that $v(h) \leq 16$. Does the same number of edges up to complementation for the $k$-element subsets, $k=8,9$ and 10, suffice for the equality of hypergraphs up to complementation? 


\subsection{Monomorphic decomposition of hypergraphs}

We present in this section a notion of monomorphic decomposition of a uniform hypergraph. This notion was introduced in [24]. Due to the introduction of an equivalence relation previously considered in [21] and developed in [20], our presentation is simpler. Let $\mathcal{H}:=(V, \mathcal{E})$ be a $h$-uniform hypergraph. Let $F \subseteq V$, we say that $\mathcal{H}$ is $F$-constant or that $V \backslash F$ is a constant block if $\mathcal{H}(\bar{A})=\mathcal{H}\left(A^{\prime}\right)$ for every $A, A^{\prime} \in[V]^{h}$ such that $A \cap F=A^{\prime} \cap F$. We say that $\mathcal{H}$ is almost-constant if it is $F$-constant for some finite subset $F$.

Example 1. A 2-uniform hypergraph is simply a (undirected) graph. If $G:=(V, \mathcal{E})$ is a graph then a subset $B$ of $V$ is a constant block if and only if it satisfies the two conditions:

1. $B$ is either a clique or an independent of $G$;

2. $B$ is an autonomous subset of $G$, that is for every $y \in V \backslash B$ and every $x, x^{\prime} \in B,\{y, x\} \in \mathcal{E} \Leftrightarrow\left\{y, x^{\prime}\right\} \in \mathcal{E}$.

Similarly, if $\mathcal{H}:=(V, \mathcal{E})$ is a h-uniform hypergraph $(h \geq 1)$, a subset $B$ of $V$ is a constant block if and only if it satisfies the two conditions:

1. $\mathcal{H}_{\uparrow B}$ is either complete (i.e. $[B]^{h} \subseteq \mathcal{E}$ ) or empty (i.e. $[B]^{h} \cap \mathcal{E}=\emptyset$ );

2. For every $k \leq h, K \in[V \backslash B]^{k}$ and $X, X^{\prime} \in[B]^{h-k}, X \cup K \in \mathcal{E} \Leftrightarrow$ $X^{\prime} \cup K \in \mathcal{E}$.

A monomorphic decomposition of a $h$-uniform hypergraph $\mathcal{H}$ is a partition of $V$ into constant blocks.

Lemma 1. A partition $\mathcal{P}$ of $V$ is a monomorphic decomposition if $\mathcal{H}(A)=$ $\mathcal{H}\left(A^{\prime}\right)$ for every $A, A^{\prime} \in[V]^{h}$ such that $|A \cap B|=\left|A^{\prime} \cap B\right|$ for every block $B \in \mathcal{P}$.

This is essentially Lemma 2.9 p.13 of [24].

Let $x, y \in V$. We set $x \equiv \mathcal{H} y$ if

$$
\mathcal{H}(K \cup\{x\})=\mathcal{H}(K \cup\{y\})
$$

for every $K \in[V \backslash\{x, y\}]^{h-1}$.

Proposition 3. The relation $\equiv_{\mathcal{H}}$ is an equivalence relation on $V$. The blocks of this equivalence are constant. They form a monomorphic decomposition of $\mathcal{H}$ and every monomorphic decomposition is finer.

Except for the introduction of the equivalence relation, this is essentially Lemma 2.11 and Proposition 2.12 p. 14 of [24]. We give an outline of the proof below. 
Proof. First, $\equiv_{\mathcal{H}}$ is an equivalence relation. For that it suffices to check that it is transitive. Let $x, y, z \in V$ with $x \equiv_{\mathcal{H}} y$ and $y \equiv_{\mathcal{H}} z$. We check that $x \equiv_{\mathcal{H}}$ $z$. We may suppose these elements pairwise distinct. Let $K \in[V \backslash\{x, z\}]^{h-1}$.

Case 1. $y \notin K$. In this case $\mathcal{H}(K \cup\{x\})=\mathcal{H}(K \cup\{y\})$ and $\mathcal{H}(K \cup\{y\})=$ $\mathcal{H}(K \cup\{z\})$. Hence $\mathcal{H}(K \cup\{x\}=\mathcal{H}(K \cup\{z\})$. Thus $x \equiv \mathcal{H} z$.

Case 2. $y \in K$. Set $K^{\prime}:=(K \backslash\{y\}) \cup\{z\}$. Since $x \equiv \mathcal{H} y$, we have $\mathcal{H}\left(K^{\prime} \cup\right.$ $\{x\})=\mathcal{H}\left(K^{\prime} \cup\{y\}\right)=\mathcal{H}(K \cup\{z\})$. Similarly, setting $K^{\prime \prime}:=(K \backslash\{y\}) \cup\{x\}$ then, since $y \equiv \mathcal{H} z$, we have $\mathcal{H}(K \cup\{x\})=\mathcal{H}\left(K^{\prime \prime} \cup\{y\}\right)=\mathcal{H}\left(K^{\prime \prime} \cup\{z\}\right)$. Since $K^{\prime} \cup\{x\}=K^{\prime \prime} \cup\{z\}$, we have $\mathcal{H}(K \cup\{x\})=\mathcal{H}(K \cup\{z\})$ and thus $x \equiv \mathcal{H} z$ as claimed. Next, the blocks of this equivalence are constant. Let $C$ be a block of $\equiv \mathcal{H}$. We prove that $\mathcal{H}(A)=\mathcal{H}\left(A^{\prime}\right)$ for every $A, A^{\prime} \in[V]^{h}$ such that $A \backslash C=A^{\prime} \backslash C$. Let $\ell:=\left|A \backslash A^{\prime}\right|$. If $\ell=0, A=A^{\prime}$, there is nothing to prove. If $\ell=1$, then $A=\{x\} \cup\left(A \cap A^{\prime}\right)$ and $A^{\prime}=\{y\} \cup\left(A \cap A^{\prime}\right)$, with $x, y \in C$; in this case $\mathcal{H}(A)=\mathcal{H}\left(A^{\prime}\right)$ since $x \equiv_{\mathcal{H}} y$. If $\ell>1$, set $K:=A \backslash C$ and $k:=|K|$. We may find a sequence of $(h-k)$-element subsets of $C$, say $A_{0}, \ldots A_{i}, \ldots, A_{r}$ such that $A_{0}=A \cap C, A_{r}=A^{\prime} \cap C$ and the symmetric difference of $A_{i}$ and $A_{i+1}$ is 0 or 1 . From the case $\ell=1$ we have $\mathcal{H}\left(A_{i} \cup K\right)=\mathcal{H}\left(A_{i+1} \cup K\right)$ for $i<r$. Hence $\mathcal{H}(A)=\mathcal{H}\left(A^{\prime}\right)$. Since these blocks are constant, they form a monomorphic decomposition. To conclude that every other monomorphic decomposition is finer, note that the elements of a constant block are pairwise equivalent w.r.t. $\equiv_{\mathcal{H}}$ hence contained into a block of this equivalence.

We call components the blocks of the equivalence relation $\equiv \mathcal{H}$. Note that the equivalence relations $\equiv_{\mathcal{H}}$ and $\equiv_{\overline{\mathcal{H}}}$ coincide and also that if $\mathcal{H}^{\prime}$ in another h-uniform hypergraph, every isomorphism of $\mathcal{H}$ onto $\mathcal{H}^{\prime}$ will transform $\equiv_{\mathcal{H}}$ into $\equiv_{\mathcal{H}^{\prime}}$, thus carrying the components of $\mathcal{H}$ onto the components of $\mathcal{H}^{\prime}$.

Let us recall Fraïssé's theorem on almost constant hypergraphs [12], this result is a consequence of the infinite form of Ramsey's theorem.

Theorem 8. Let $h$ be a non-negative integer, $\mathcal{H}$ be a h-uniform hypergraph on an infinite set $V$ and $F$ be a finite subset of $V$. Then there is an infinite subset $V^{\prime}$ of $V$ such that $\mathcal{H}_{\uparrow V^{\prime}}$ is $F$-constant.

Let $\psi(h):=h+2^{t}$ where $t$ is the largest integer $t^{\prime}$ such that $2^{t^{\prime}} \leq h$, that is $\psi(h)=h+2^{\left\lfloor\log _{2} h\right\rfloor}$.

Theorem 9. Let $\mathcal{H}$ and $\mathcal{H}^{\prime}$ be two h-uniform hypergraphs on the same set $V$ of vertices. Suppose that

1) $\mathcal{H}$ and $\mathcal{H}^{\prime}$ are $F$-constant for some $F \subseteq V$,

2) $|V \backslash F| \geq h$,

3) $\mathcal{H}$ and $\overline{\mathcal{H}}^{\prime}$ are $\psi(h)$-hypomorphic up to complementation. 
Then $\mathcal{H}=\mathcal{H}^{\prime}$ or $\mathcal{H}=\overline{\mathcal{H}^{\prime}}$.

Proof. We may suppose that $\mathcal{H}$ and $\mathcal{H}^{\prime}$ coincide on $V \backslash F$ (otherwise replace $\mathcal{H}^{\prime}$ by $\overline{\mathcal{H}^{\prime}}$ ) and for example that there is no hyperedge in $V \backslash F$. We prove that $\mathcal{H}=\mathcal{H}^{\prime}$. For that, we prove by induction on $\ell$ that:

$$
\mathcal{H}(A)=\mathcal{H}^{\prime}(A)
$$

for every $A \in[V]^{h}$ such that $|A \cap F| \leq \ell$.

If $\ell:=0$ then since $\mathcal{H}$ and $\mathcal{H}^{\prime}$ coincide on $V \backslash F$, Equation (2) holds.

Suppose that $\ell \geq 1$. Let $A \in[V]^{h}$ such that $|F \cap A| \leq \ell$. Let $F_{0}:=A \cap F$. If $\left|F_{0}\right|<\ell$ then (2) holds by induction. Suppose that $\left|F_{0}\right|=\ell$. Let $S$ and $K:=A \cup S$ where $S \subseteq V \backslash(F \cup A),|S|=s$, and $k:=h+s=\psi(h)$. Let $\mathcal{H}_{0}:=\mathcal{H}_{\uparrow K}, \mathcal{H}_{0}^{\prime}:=\mathcal{H}_{\uparrow K}^{\prime}$. We have $K \in[V]^{k}$ and $F_{0}=K \cap F$.

Since $\mathcal{H}$ and $\mathcal{H}^{\prime}$ are $F$-constant, the $h$-uniform hypergraphs $\mathcal{H}_{0}$ and $\mathcal{H}_{0}^{\prime}$ are $F_{0}$-constant.

The set $\mathcal{E}\left(\mathcal{H}_{0}\right)$ of hyperedges of $\mathcal{H}_{0}$ is the disjoint union of $\mathcal{E}_{<\ell}\left(\mathcal{H}_{0}\right):=$ $\left\{A^{\prime} \in \mathcal{E}\left(\mathcal{H}_{0}\right):\left|A^{\prime} \cap F\right|<\ell\right\}$ and $\mathcal{E}_{=\ell}\left(\mathcal{H}_{0}\right):=\left\{A^{\prime} \in \mathcal{E}\left(\mathcal{H}_{0}\right):\left|A^{\prime} \cap F\right|=\ell\right\}:$

$$
\mathcal{E}\left(\mathcal{H}_{0}\right)=\mathcal{E}_{<\ell}\left(\mathcal{H}_{0}\right) \cup \mathcal{E}_{=\ell}\left(\mathcal{H}_{0}\right)
$$

Similarly, $\mathcal{H}_{0}^{\prime}$ decomposes into $\mathcal{E}_{<\ell}\left(\mathcal{H}_{0}^{\prime}\right)$ and $\mathcal{E}_{=\ell}\left(\mathcal{H}_{0}^{\prime}\right)$ :

$$
\mathcal{E}\left(\mathcal{H}_{0}^{\prime}\right)=\mathcal{E}_{<\ell}\left(\mathcal{H}_{0}^{\prime}\right) \cup \mathcal{E}_{=\ell}\left(\mathcal{H}_{0}^{\prime}\right)
$$

By induction hypothesis,

$$
\mathcal{E}_{<\ell}\left(\mathcal{H}_{0}\right)=\mathcal{E}_{<\ell}\left(\mathcal{H}_{0}^{\prime}\right)
$$

Claim 1. If $\mathcal{H}_{0} \simeq \mathcal{H}_{0}^{\prime}$ then $\mathcal{H}_{0}=\mathcal{H}_{0}^{\prime}$ and hence (2) holds.

Indeed, we have $\left|\mathcal{E}\left(\mathcal{H}_{0}\right)\right|=\left|\mathcal{E}\left(\mathcal{H}_{0}^{\prime}\right)\right|$. From (3), (4) and (5), it follows that $\left|\mathcal{E}_{=\ell}\left(\mathcal{H}_{0}\right)\right|=\left|\mathcal{E}_{=\ell}\left(\mathcal{H}_{0}^{\prime}\right)\right|$. Since $\mathcal{H}_{0}$ is $F_{0}$-constant, then $\mathcal{E}_{=\ell}\left(\mathcal{H}_{0}\right)=\emptyset$ or $\mathcal{E}_{=\ell}\left(\mathcal{H}_{0}\right)=\left\{F_{0} \cup I: I \in[S]^{h-l}\right\}$. The same holds for $\mathcal{H}^{\prime}$, hence $\mathcal{E}_{=\ell}\left(\mathcal{H}_{0}\right)=$ $\mathcal{E}_{=\ell}\left(\mathcal{H}_{0}^{\prime}\right)$.

Thus $\mathcal{E}\left(\mathcal{H}_{0}\right)=\mathcal{E}\left(\mathcal{H}_{0}^{\prime}\right)$ proving $\mathcal{H}_{0}=\mathcal{H}_{0}^{\prime}$.

To conclude, we prove that $\mathcal{H}_{0} \not \overline{\mathcal{H}_{0}^{\prime}}$.

Case 1. $k \geq 2 l+1$. Let $C:=K \backslash F_{0}$. We have $|C|>\left|F_{0}\right|$. Since $\mathcal{H}_{0}$ is $F_{0}$-constant, $C$ is a constant block. According to Proposition 3, $C$ is included into a component $D$ of $\mathcal{H}_{0}$. Similarly $C$ is included into a component $D^{\prime}$ of $\mathcal{H}_{0}^{\prime}$. Since $|C|>\left|F_{0}\right|$, all components of $\mathcal{H}_{0}$ distinct of $D$ have a cardinality strictly smaller than $|D|$. The same with $D^{\prime}$ the component of $\overline{\mathcal{H}_{0}^{\prime}}$ containing $C$. An isomorphism from $\mathcal{H}_{0}$ onto $\overline{\mathcal{H}_{0}^{\prime}}$ will map $D$ onto $D^{\prime}$. But there is no 
hyperedge of $\mathcal{H}$ in $D$ whereas every $h$-element subset of $D$ is a hyperedge of $\overline{\mathcal{H}_{0}^{\prime}}$. Contradiction.

Case 2. $k-\ell \leq \ell$. By the induction hypothesis for $\ell^{\prime}<\ell$ and the fact that $\mathcal{H}_{0} \simeq \overline{\mathcal{H}_{0}^{\prime}}$, we have:

$$
2 .\left|\mathcal{E}_{<\ell}\left(\mathcal{H}_{0}\right)\right|+\left(\begin{array}{l}
k-l \\
h-l
\end{array}\right)=\left(\begin{array}{l}
k \\
h
\end{array}\right) .
$$

Recall that $k=h+s=\psi(h)$ with $s=2^{t}$ where $t$ is the largest integer $t^{\prime}$ such that $2^{t^{\prime}} \leq h$. Then $s \leq h<2^{t+1}$. We have $h=\sum_{i=0}^{t} a_{i} 2^{i}$ with $a_{i} \in\{0,1\}$, $a_{t}=1, k=\sum_{i=0}^{t-1} a_{i} 2^{i}+2^{t+1}$. From Corollary $1,\left(\begin{array}{c}k \\ h\end{array}\right)$ is even. Since $\frac{k}{2} \leq \ell \leq h$ then $\left(\begin{array}{l}k-\ell \\ h-\ell\end{array}\right)$ is odd. Indeed, $\ell \geq s$, thus $h-l<2^{t}$, so $h-l=\sum_{i=0}^{t-1} c_{i} 2^{i}$ with $c_{i} \in\{0,1\}$, and thus $k-l=\sum_{i=0}^{t-1} c_{i} 2^{i}+2^{t}$. Then, by Corollary $1,\left(\begin{array}{c}k-\ell \\ h-\ell\end{array}\right)$ is odd. The facts that $\left(\begin{array}{l}k \\ h\end{array}\right)$ is even and $\left(\begin{array}{c}k-\ell \\ h-\ell\end{array}\right)$ is odd contradict Equation (6).

\section{Proof of Theorem 1}

Suppose that for some $h$ the statement is false.

Claim 2. There are two h-uniform hypergraphs $\mathcal{H}^{(i)}, i=1,2$, on $\mathbb{N}^{*}$ which are not equal up to complementation but are $k$-hypomorphic up to complementation for every $k \in \mathbb{N}$.

Proof of Claim 2. We use a compactness argument. Let $\mathcal{H}\left(h, \mathbb{N}^{*}\right)$ be the set of $h$-uniform hypergraphs with domain $\mathbb{N}^{*}:=\mathbb{N} \backslash\{0\}$. Essentially, this is the power set $\wp\left(\left[\mathbb{N}^{*}\right]^{h}\right)$. Once equipped with the product topology, the power set is a compact space, hence we may view $\mathcal{H}\left(h, \mathbb{N}^{*}\right)$ as a compact space and also the product space $\mathcal{H}^{2}\left(h, \mathbb{N}^{*}\right):=\mathcal{H}\left(h, \mathbb{N}^{*}\right) \times \mathcal{H}\left(h, \mathbb{N}^{*}\right)$ of pairs $\left(\mathcal{H}^{(1)}, \mathcal{H}^{(2)}\right)$ of $h$ uniform hypergraph with domain $\mathbb{N}^{*}$. Let $\mathcal{B}$ be the set of pairs $\left(\mathcal{H}^{(1)}, \mathcal{H}^{(2)}\right) \in$ $\mathcal{H}^{2}\left(h, \mathbb{N}^{*}\right)$ which are $k$-hypomorphic up to complementation for every $k \in \mathbb{N}$ but whose restrictions to $\{1, \ldots, 2 h\}$ are not equal up to complementation. To prove our claim, it suffices to prove that $\mathcal{B}$ is non-empty. For that, we prove that this set is the intersection of a decreasing sequence of non-empty closed subsets of $\mathcal{H}^{2}\left(h, \mathbb{N}^{*}\right)$. The compactness of this set ensures the nonemptyness of $\mathcal{B}$. For every integer $k$, let $\mathcal{B}_{k}$ be the set of pairs $\left(\mathcal{H}^{(1)}, \mathcal{H}^{(2)}\right) \in$ $\mathcal{H}^{2}\left(h, \mathbb{N}^{*}\right)$ such that the restrictions to $\{1, \ldots, 2 k-1\}$ are $k$-hypomorphic up to complementation and the restrictions to $\{1, \ldots, 2 h\}$ are not equal up to complementation. Trivially, we have $\mathcal{B}=\bigcap_{k \in \mathbb{N}} \mathcal{B}_{k}$. Due to Proposition 1 , we have $\mathcal{B}_{k+1} \subseteq \mathcal{B}_{k}$. Since the set of restrictions to $\{1, \ldots, 2 k-1\}$ of members of $\mathcal{H}\left(h, \mathbb{N}^{*}\right)$ is finite, the $\mathcal{B}_{k}$ 's are closed. To conclude, it suffices to observe that they are non-empty. Let $k \in \mathbb{N}$. Since the statement of the theorem is assumed to be false, then for every $t, k \leq t$, there is some $t^{\prime} \geq t$ 
and two hypergraphs $\mathcal{H}_{k}^{(i)}(i=1,2)$ on $V_{t^{\prime}}:=\left\{1, \ldots, t^{\prime}\right\}$ which are not equal up to complementation, but are $k$-hypomorphic up to complementation. We can assume that $\mathcal{H}_{k}^{(i)}(i=1,2)$ are not equal up to complementation on $\{1, \ldots, l\}$ where $l \leq 2 h$ (otherwise, consider the hypergraphs obtained via a permutation transforming a subset $L$ of $V_{t^{\prime}}$ on which they are not equal up to complementation into $\{1, \ldots, l\})$. Let $t:=2 k-1$ and $t^{\prime}$ corresponding to $t$. Let $\mathcal{H}^{(i)}$ be a $h$-uniform hypergraph on $\mathbb{N}^{*}$ which extends $\mathcal{H}_{k}^{(i)}$ arbitrarily. We have $\left(\mathcal{H}^{(1)}, \mathcal{H}^{(2)}\right) \in \mathcal{B}_{k}$.

A contradiction is obtained through Theorem 8 . Indeed, there is a subset $F$ with at most $2 h$ elements on which $\mathcal{H}^{1}$ and $\mathcal{H}^{2}$ are not equal up to complementation. According to Theorem 8, applied twice, there is an infinite subset $V^{\prime}$ of $V$ containing $F$ such that $\mathcal{H}_{\uparrow V^{\prime}}^{1}$ and $\mathcal{H}_{\uparrow V^{\prime}}^{2}$ are $F$-constant. These two hypergraphs are $k$-hypomorphic up to complementation for all $k$. This contradicts Theorem 9 .

\section{Proof of Theorem 2}

Let $\varphi(h):=h+2^{\left\lfloor\log _{2} h\right\rfloor}-1$. For example $\varphi(h)=2 h-1$ if $h$ is a power of 2 . Theorem 9 asserts that $s(h) \leq \psi(h)=\varphi(h)+1$. Theorem 10 below asserts that $s(h) \geq \varphi(h)+1$. Thus $s(h)=h+2^{\left\lfloor\log _{2} h\right\rfloor}$. This proves (1) of Theorem 2. Theorem 3.1 and Proposition 4.1 of [9] assert that $s(2)=4$. We obtain (2) of Theorem 2 with Theorem 7.

Theorem 10. Let $h$ be an integer. There are two $h$-uniform infinite hypergraphs $\mathcal{H}$ and $\mathcal{H}^{\prime}$ on the same domain such that $\mathcal{H} \neq \mathcal{H}^{\prime}$ and $\mathcal{H} \neq \overline{\mathcal{H}^{\prime}}$, but $\mathcal{H}$ and $\mathcal{H}^{\prime}$ are $k$-hypomorphic up to complementation for all $k \leq \varphi(h)$. These two hypergraphs are $F$-constant for some subset $F$ of cardinality at most $h$.

Proof. Let $V$ be an infinite set, and $F \subseteq V$ having $r$ elements with $2 \leq r \leq h$.

Let $\wp^{\star}(F):=\wp(F) \backslash\{\emptyset, F\}$ be the set of proper subsets of $F$. Suppose that $\left\{\mathcal{A}, \mathcal{A}^{\prime}\right\}$ is a partition of $\wp^{\star}(F)$ into two blocks and that $\varphi$ is a permutation of $F$ such that for all $X \in \wp^{\star}(F)$ :

$$
X \in \mathcal{A} \Longleftrightarrow \varphi(X) \in \mathcal{A}^{\prime}
$$

We define two hypergraphs $\mathcal{H}$ and $\mathcal{H}^{\prime}$ as follows:

$\mathcal{H}:=(V, \mathcal{E})$ where $\mathcal{E}:=\left\{A \in[V]^{h}: A \cap F \in \mathcal{A}\right\}$ and $\mathcal{H}^{\prime}:=\left(V, \mathcal{E}^{\prime}\right)$ where $\mathcal{E}^{\prime}:=\mathcal{E} \cup\left\{A \in[V]^{h}: F \subseteq A\right\}$.

Claim 3. $\mathcal{H} \neq \mathcal{H}^{\prime}$ and $\mathcal{H} \neq \overline{\mathcal{H}^{\prime}}$.

Proof. Every $h$-element subset of $V$ containing $F$ belongs to $\mathcal{H}^{\prime}$, whereas none of these sets is in $\mathcal{H}$. This proves that $\mathcal{H} \neq \mathcal{H}^{\prime}$. Every $h$-element subset 
of $V \backslash F$ belongs to $\overline{\mathcal{H}^{\prime}}$, none of these sets is in $\mathcal{H}$ proving $\mathcal{H} \neq \overline{\mathcal{H}^{\prime}}$.

Claim 4. $\mathcal{H}$ and $\mathcal{H}^{\prime}$ are $k$-hypomorphic up to complementation for $k \leq$ $h+r-1$.

Proof of Claim 4. Since $V$ is infinite, Proposition 1 applies. Hence it suffices to prove this property for $k=h+r-1$. Let $K \subseteq V$, with $|K|=h+r-1$.

Case 1. $F \nsubseteq K$. Then clearly $\mathcal{H}_{\uparrow K}=\mathcal{H}^{\prime}{ }_{\uparrow K}$.

Case 2. $F \subseteq K$. Let $\bar{\varphi}$ the map from $K$ into $K$ defined by $\bar{\varphi}(x)=\varphi(x)$ if $x \in F$, and $\bar{\varphi}(x)=x$ if $x \in K \backslash F$. Clearly, $\bar{\varphi}$ is a permutation of $K$. We will show that $\bar{\varphi}$ is an isomorphism from $\mathcal{H}_{\uparrow K}$ onto $\overline{\mathcal{H}^{\prime}{ }_{\uparrow K}}$. Let $A \in[K]^{h}$, we will check the equivalence:

$$
A \in \mathcal{E}_{\uparrow K} \Longleftrightarrow \bar{\varphi}(A) \in \overline{\mathcal{E}^{\prime}\lceil K}
$$

Note that $A \in \mathcal{E}_{\uparrow K} \Longleftrightarrow A \cap F \in \mathcal{A}$. Also, note that $A \cap F \in \mathcal{A} \Longleftrightarrow$ $\varphi(A \cap F) \in \mathcal{A}^{\prime}$. Since $\bar{\varphi}(A) \cap F=\varphi(A \cap F)$ we have

$$
A \in \mathcal{E}_{\uparrow K} \Longleftrightarrow \bar{\varphi}(A) \cap F \in \mathcal{A}^{\prime}
$$

Now, $\bar{\varphi}(A) \in \mathcal{E}^{\prime}{ }_{\mid K} \Longleftrightarrow \bar{\varphi}(A) \cap F \in \mathcal{A}$ or $F \subseteq A$, that is:

$$
\bar{\varphi}(A) \notin \mathcal{E}^{\prime}{ }_{\lceil K} \Longleftrightarrow \bar{\varphi}(A) \cap F \notin \mathcal{A} \text { and } F \nsubseteq A .
$$

To check (8) it suffices to check

$$
\bar{\varphi}(A) \cap F \in \mathcal{A}^{\prime} \Longleftrightarrow \bar{\varphi}(A) \cap F \notin \mathcal{A} \text { and } F \nsubseteq A .
$$

$\bar{\varphi}(A) \cap F \in \mathcal{A}^{\prime} \Rightarrow \bar{\varphi}(A) \cap F \notin \mathcal{A}$ (because $\left\{\mathcal{A}, \mathcal{A}^{\prime}\right\}$ is a partition), and $F \nsubseteq \bar{\varphi}(A)$. Since $\bar{\varphi}(F)=F, F \nsubseteq A$. Conversely, since $F \nsubseteq A, F \cap A \neq F$. From $|F|=r \leq h=|A|, F \subseteq K$ and $|K|=h+r-1$, we have $F \cap A \neq \emptyset$. Then $F \cap A$ is a proper subset of $F$, thus $\bar{\varphi}(A) \cap F$ is a proper subset of $F$. Since $\bar{\varphi}(A) \cap F \notin \mathcal{A}, \bar{\varphi}(A) \cap F \in \mathcal{A}^{\prime}$.

Claim 5. Let $F$ be a r-element subset of $V, r \geq 2$. Then there is a partition $\left\{\mathcal{A}, \mathcal{A}^{\prime}\right\}$ of $\wp^{\star}(F)$ into two blocks and a permutation $\varphi$ of $F$ satisfying Equation (7) if and only if $r$ is a power of 2 .

Proof of Claim 5. If there is a partition $\left\{\mathcal{A}, \mathcal{A}^{\prime}\right\}$ of $\wp^{\star}(F)$ into two blocks and a permutation $\varphi$ of $F$ satisfying Equation (7), then for each integer $k$, with $1 \leq k \leq r-1$, the number of $k$-element subsets of $F$ is even, thus $\left(\begin{array}{l}r \\ k\end{array}\right)$ is even for all $k \in\{1, \ldots, r-1\}$. Then, by Corollary $1, r$ is a power of 2 .

Conversely, if $r$ is a power of 2 , with $r \geq 2$, we can find a permutation $\varphi$ of a $r$-element set $F$ and a partition of $\rho^{\star}(F)$ into two classes $\mathcal{A}, \mathcal{A}^{\prime}$ such that for each proper subset $K$ of $F, \bar{\varphi}(K)$ and $K$ are not in the same class 
(where $\bar{\varphi}(X)=\{\varphi(x): x \in X\}$ ). Take for $\varphi$ a circular permutation of $F$. Fix an integer $k$. Let $\bar{\varphi}$ be the induced permutation on $[F]^{k}$. We have $\bar{\varphi}^{r}=\overline{\varphi^{r}}=i d_{{ }_{\star}(F)}$. Then the order of $\bar{\varphi}$ divides $r$, therefore it is of the form $2^{r^{\prime}}$. It is easy to see that $\bar{\varphi}^{r}$ has no fixed point. Decompose $\bar{\varphi}$ in cycles, the order of each cycle divides $2^{r}$, so is even. So each cycle is not trivial with even order. We say that two subsets of $F$ of size $k$ are equivalent if we pass from one to the other by some $\bar{\varphi}^{s}$ with $s$ even. This gives a partition $\left\{\mathcal{A}, \mathcal{A}^{\prime}\right\}$ of $\wp^{\star}(F)$ into two blocks satisfying Equation (7).

\section{Possible generalizations}

Various kind of isomorphy have been considered for hypergraphs e.g [2]. Here we consider the following notion. Let $W$ be a set and $\mathfrak{G}$ a subgroup of the group $\mathfrak{S}(W)$ of permutations of $W$. Let $h$ be an integer. A $h$-uniform hypergraph valued by $W$ is a pair $\mathcal{H}:=(V, c)$ where $c$ is a map from $[V]^{h}$ into $W$. Let $\mathcal{H}:=(V, c)$ and $\mathcal{H}^{\prime}:=\left(V^{\prime}, c^{\prime}\right)$ be two $h$-uniform hypergraphs valued by $W$; a map $f: V \rightarrow V^{\prime}$ is an isomorphism up to $\mathfrak{G}$ if there is some $\sigma \in \mathfrak{G}$ such that $c^{\prime} \circ \bar{f}=\sigma \circ c$ (where $\bar{f}$ is the extension of $f$ to $[V]^{h}$ ). We say that $\mathcal{H}$ and $\mathcal{H}^{\prime}$ are equal up to $\mathfrak{G}$ if $V=V^{\prime}$ and the identity map is an isomorphism up to $\mathfrak{G}$ of $\mathcal{H}$ onto $\mathcal{H}^{\prime}$ (that is $c^{\prime}=\sigma \circ c$ for some $\sigma \in \mathfrak{G}$ ).

\subsection{Equality and isomorphy up to a permutation group}

It is natural to ask if there are two non-negative integers $k$ and $t, k \leq t$, such that two $h$-uniform hypergraphs $\mathcal{H}$ and $\mathcal{H}^{\prime}$ on the same set $V$ of vertices, $|V| \geq t$, and valued by $W$, are equal up to $\mathfrak{G}$ whenever $\mathcal{H}$ and $\mathcal{H}^{\prime}$ are $k$-hypomorphic up to $\mathfrak{G}$.

The answer is negative in general. Here is an example for $h=2, W=$ $\{0,1,2\}$ and $\mathfrak{G}:=\mathfrak{S}(W)$ the symmetric group on $W$.

Take $V:=\mathbb{N}, \mathcal{H}:=(V, c)$ where $c(\{0,1\})=c(\{1,2\})=0, c(\{0,2\})=$ $1, c(\{x, y\})=2$ for all other pairs and $\mathcal{H}^{\prime}:=\left(V, c^{\prime}\right)$ where $c^{\prime}(\{0,1\})=$ $c^{\prime}(\{0,2\})=0, c^{\prime}(\{1,2\})=1$ and $c^{\prime}(\{x, y\})=2$ for all other pairs.

Problem 3. Is it true that there are two non-negative integers $k$ and $t, k \leq$ $t$, such that two graphs $\mathcal{G}$ and $\mathcal{G}^{\prime}$ on the same set $V$ of vertices, $|V| \geq t$ and valued by $W$, are isomorphic up to $\mathfrak{S}(W)$ whenever they are $k$-hypomorphic up to $\mathfrak{S}(W)$ ?

The integers $k$ and $t$ if they exist will depend upon the cardinality of $W$. We give an example of two labelled graphs showing that $k$ must be at least 
$\frac{2}{3}|W|-2$. This example, inspired of [23], somewhat codes a cylinder and a Moebius band.

Let $n \in \mathbb{N}, n \geq 3, W:=(\mathbb{Z} / n \mathbb{Z} \times \mathbb{Z} / 3 \mathbb{Z}) \cup\{0,1\}$ and $V:=\mathbb{Z} / n \mathbb{Z} \times \mathbb{Z} / 2 \mathbb{Z}$. Let $c:[V]^{2} \rightarrow W$ defined by: $c(\{(i, 0),(i, 1)\}):=1, c(\{(i, 0),(i+1,0)\})=$ $c(\{(i, 1),(i+1,1)\}):=(i, 0), c(\{(i, 0),(i+1,1)\}):=(i, 1), c(\{(i, 1),(i+$ $1,0)\}):=(i, 2)$ and $c(\{u, v\})=0$ for all other pairs. Let $c^{\prime}:[V]^{2} \rightarrow W$ be obtained from $c$ by changing the values on the pairs $\{(0, i),(1, j)\}$ with $i, j \in\{0,1\}$ so that $c^{\prime}(\{(0,0),(1,1)\})=c^{\prime}(\{(0,1),(1,0)\})=(0,0), c^{\prime}(\{(0,1)$, $(1,1)\})=(0,2)$, and $c^{\prime}(\{(0,0),(1,0)\})=(0,1)$.

Lemma 2. The valued graphs $G:=(V, c)$ and $G^{\prime}:=\left(V, c^{\prime}\right)$ are not isomorphic up to $\mathfrak{S}(W)$ but their restrictions to every proper subset of $V$ are isomorphic up to $\mathfrak{S}(W)$.

If we set $k:=2 n-1$ then since $|W|=3 n+2$ and $|V|=2 n$, we have $k<\frac{2}{3}|W|-2\left(=2 n-\frac{2}{3}\right)$, the claim ensures that this values of $k$ is too small to yield an isomorphy up to a permutation group.

Proof of Lemma 2. 1) $G$ and $G^{\prime}$ are not isomorphic up to $\mathfrak{S}(W)$. Indeed, associate to $G=(V, c)$ the graph $G_{c}$ whose vertex set is the set ${ }^{2} V$ of directed pairs $(x, y)$ of distinct elements of $V$ and edges are pairs $\left\{e, e^{\prime}\right\}$ with $e=(x, y), e^{\prime}=\left(x^{\prime}, y^{\prime}\right)$ such that $c(\{x, y\})=c\left(\left\{x^{\prime}, y^{\prime}\right\}\right), c\left(\left\{x, x^{\prime}\right\}\right)=$ $c\left(\left\{y, y^{\prime}\right\}\right) \neq c(\{x, y\}), c\left(\left\{x, y^{\prime}\right\}\right) \neq c\left(\left\{y, x^{\prime}\right\}\right), c\left(\left\{x, y^{\prime}\right\}\right)$ and $c\left(\left\{y, x^{\prime}\right\}\right)$ distinct from $c(\{x, y\})$ and $c\left(\left\{x, x^{\prime}\right\}\right)$. Then, observe that if a directed pair $e:=(x, y)$ is not an isolated vertex in this graph then $\{x, y\} \in\{\{(i, 0),(i, 1)\}$, $\{(i, 0),(i+1,0)\},\{(i, 1),(i+1,1)\}\}$. Next, observe that $G_{c}$ contains exactly two cycles of length $n$; one made of the vertices $((i, 0),(i, 1)), i \in \mathbb{Z} / n \mathbb{Z}$, the other made of vertices $((i, 1),(i, 0))$ for $i \in \mathbb{Z} / n \mathbb{Z}$. Let $G_{c^{\prime}}^{\prime}$ be the graph defined by the same way. This graph contains a cycle of length $2 n$, made of vertices $((i, j),(i, j+1)),(i, j) \in \mathbb{Z} / n \mathbb{Z} \times \mathbb{Z} / 2 \mathbb{Z}$, the cycle being enumerated as $((0,0),(0,1)),((1,1),(1,0)),((2,1),(2,0)), \ldots,((n-1,1),(n-1,0))$, $((0,1),(0,0)),((1,0),(1,1)),((2,0),(2,1)), \ldots,((n-1,0),(n-1,1))$. There is no shorter cycle. Suppose that $G$ and $G^{\prime}$ are isomorphic up to $\mathfrak{S}(W)$ via some map $f$ and some permutation of $W$. The map $f$ induces an isomorphism from $G_{c}$ onto $G_{c^{\prime}}^{\prime}$ hence a $n$-cycle of $G_{c}$ is sent onto an $n$-cycle of $G_{c^{\prime}}^{\prime}$, but there are none. A contradiction.

2) For every proper subset $K$ of $V$ the restrictions $G_{\lceil K}$ and $G_{\lceil K}^{\prime}$ are isomorphic up to $\mathfrak{S}(W)$. First, suppose that $K$ is disjoint from one of the sets $\{(0,0),(0,1)\},\{(1,0),(1,1)\}$ then the two valued graphs are identical hence isomorphic. Next, suppose that $K$ is not disjoint from these sets but does not contain some element of $L:=\{(0,0),(0,1),(1,0),(1,1)\}$. We claim that in this case, the identity on $K$ and some permutation $\sigma$ of $W$ form an isomorphism up to $\mathfrak{S}(W)$ from $G_{\uparrow K}$ onto $G_{\uparrow K}^{\prime}$. Suppose for an example 
that $(0,1) \notin K$ holds, thus $(0,0) \in K$. In this case, set $\sigma$ the permutation of $W$ such that $\sigma((0,0))=(0,1), \sigma((0,1))=(0,0)$ and $\sigma(u)=u$ for every $u \in W \backslash\{(0,0),(0,1)\}$. Finally, suppose that $L \subseteq K$. Let $i_{1}$ be the largest integer such that $K_{1}:=\left\{1,2, \ldots, i_{1}\right\} \times \mathbb{Z} / 2 \mathbb{Z} \subseteq K$. Since $(1,0),(1,1) \in K$, the integer $i_{1}$ is well-defined. Furthermore, $i_{1}<n-1$. Otherwise, since $(0,0)$ and $(0,1)$ belong to $K$, we would have $K=\mathbb{Z} / n \mathbb{Z} \times \mathbb{Z} / 2 \mathbb{Z}=V$. From the definition of $i_{1}$, we have $\left(i_{1}+1,0\right)$ or $\left(i_{1}+1,1\right) \notin K$. Suppose that $\left(i_{1}+1,1\right) \notin K$. The case $\left(i_{1}+1,0\right) \notin K$ will be similar. Let $f$ be the map from $K$ to $K$ which is the identity on $K \backslash K_{1}$ and which exchanges $(i, 0)$ and $(i, 1)$ on $K_{1}$ and let $\sigma$ be the permutation of $W$ such that $\sigma\left(i_{1}, 0\right)=\left(i_{1}, 2\right)$, $\sigma\left(i_{1}, 2\right)=\left(i_{1}, 0\right), \sigma(i, 1)=(i, 2), \sigma(i, 2)=(i, 1)$ for $i \in\left\{1,2, \ldots, i_{1}-1\right\}$ and $\sigma(u)=u$ for other elements of $W$.

Note that

$$
\sigma(i, j) \neq(i, j) \Rightarrow i \in\left\{1,2, \ldots, i_{1}\right\}
$$

We check that $f$ is an isomorphism with respect to $\sigma$, that is:

$$
\sigma(c(u, v))=c^{\prime}(f(u), f(v)) \text { for every } u, v \in K .
$$

If $u, v \in K \backslash K_{1}$, then $f(u)=u, f(v)=v$ and due to $(12), c^{\prime}(u, v)=\sigma(c(u, v))$ as required by (13).

Suppose that $u, v \in K_{1}$. We have $u=(i, j), v=\left(i^{\prime}, j^{\prime}\right)$ and $u^{\prime}:=$ $f(i, j)=(i, j+1), v^{\prime}:=f\left(i^{\prime}, j^{\prime}\right)=\left(i^{\prime}, j^{\prime} \dot{+} 1\right)$ where $\dot{+}$ is the sum modulo 2.

If $i=i^{\prime}, c^{\prime}\left(u^{\prime}, v^{\prime}\right)=c(u, v)=\sigma(c(u, v))$ as required by (13). If $i \neq i^{\prime}$, we may suppose $i^{\prime}=i \dot{+} 1$. There are two cases. First $j=j^{\prime}$. In this case, $c(u, v)=(i, 0), c^{\prime}\left(u^{\prime}, v^{\prime}\right)=(i, 0)=\sigma(i, 0)$ since $i \neq i_{1}$. Next $j \neq j^{\prime}$, if $u=(i, 1)$ and $v=(i+1,0)$, then $c(u, v)=(i, 2), c^{\prime}\left(u^{\prime}, v^{\prime}\right)=(i, 1)=\sigma(i, 2)$. The case $u=(i, 0)$ and $v=(i+1,1)$ is similar.

Suppose that $u \in K_{1}$ and $v \in K \backslash K_{1}$. Hence $u^{\prime}=(i, j+1)$ and $v^{\prime}=$ $v=\left(i^{\prime}, j^{\prime}\right)$. We may suppose that either $\left(i=1\right.$ and $\left.i^{\prime}=0\right)$ or $i=i_{1}$ and $i^{\prime}=i_{1}+1$. In the first case, suppose that $u=(1,1)$. If $v=(1,0)$ then $c(u, v)=(0,0)$, whereas $c^{\prime}\left(u^{\prime}, v^{\prime}\right)=(0,0)=\sigma(c(u, v))$. If $v=(0,0)$ then $c(u, v)=(0,1)$, whereas $c^{\prime}\left(u^{\prime}, v^{\prime}\right)=(0,1)=\sigma(c(u, v))$. The case $u=(0,0)$ is similar. In the second case, we have $j^{\prime}=0$. If $j=1$ then, $c(u, v)=\left(i_{1}, 2\right)$ and $c^{\prime}\left(u^{\prime}, v^{\prime}\right)=\left(i_{1}, 0\right)=\sigma(c(u, v))$. If $j=0$ then $c(u, v)=\left(i_{1}, 0\right)$ and $c^{\prime}\left(u^{\prime}, v^{\prime}\right)=\left(i_{1}, 2\right)=\sigma(c(u, v))$.

Problem 4. Find examples showing that $k \geq 2|W|$.

A special instance of Problem 3 is this. Let $n$ be a non-negative integer, suppose that $W:=\{0,1\}^{n}$ and that $\mathfrak{G}$ is the permutation group made of the identity and the involution on $W$ defined by $\bar{u}:=u+1$ where 
$1:=(1, \ldots, 1) \in W$ and the addition is modulo 2 . A valued graph $G:=(V, c)$ by $W$ identifies to a multigraph; if we define the complement of $G$ by setting $\bar{G}:=(V, \bar{c})$ where $\bar{c}(\{x, y\}):=\overline{c(\{x, y\})}$, then isomorphy up to complementation means isomorphy up to $\mathfrak{G}$. Find $k$ and $t$ such that the conclusion of Problem 3 holds. For $n=1$ this is the result of [9].

\subsection{Isomorphy of hypergraphs up to a permutation group and isomorphy of relational structures}

Associate to each valued $h$-uniform hypergraph $\mathcal{H}:=(V, c)$ valued by $W$ the pair $\widehat{\mathcal{H}}:=\left(V, E q_{c}\right)$ where $E q_{c}$ is the kernel of $c$, that is the equivalence relation defined on $[V]^{h}$ by $A E q_{c} A^{\prime}$ if $c(A)=c\left(A^{\prime}\right)$. Associate also the $2 h$ ary-structure $\widetilde{\mathcal{H}}:=\left(V, \rho_{c}\right)$ where $\rho_{c}$ is the subset of $V^{2 h}$ made of $2 h$-uples $\left(x_{1}, \ldots x_{2 h}\right)$ such that $c\left(\left\{x_{1}, \ldots x_{h}\right\}\right)=c\left(\left\{x_{h+1}, \ldots, x_{2 h}\right\}\right)$.

We have:

Lemma 3. Let $\mathfrak{G}:=\mathfrak{S}(W)$ be the symmetric group on $W$. Let $\mathcal{H}:=(V, c)$ and $\mathcal{H}^{\prime}:=\left(V^{\prime}, c^{\prime}\right)$ be two h-uniform hypergraphs valued by $W$ and a map $f: V \rightarrow V^{\prime}$. Then the following properties are equivalent:

(i) $f$ is an isomorphism up to $\mathfrak{G}$ from $\mathcal{H}$ onto $\mathcal{H}^{\prime}$;

(ii) $f$ is bijective and

$$
H E q_{c} H^{\prime} \Leftrightarrow f(H) E q_{c^{\prime}} f\left(H^{\prime}\right) \text { for all } H, H^{\prime} \in[V]^{h}
$$

(iii) $f$ is an isomorphism from $\widetilde{\mathcal{H}}$ onto $\widetilde{\mathcal{H}^{\prime}}$.

We mention that with Lemma 3 and Theorem 3, Proposition 1 is generalized as follows.

Proposition 4. Let $W$ be a set and $\mathfrak{G}$ be a subgroup of the group $\mathfrak{S}(W)$ of permutations of $W$. Let $v, k$ be non-negative integers, Let $t \leq \min (k, v-k)$ and $\mathcal{H}$ and $\mathcal{H}^{\prime}$ be two h-uniform hypergraphs, on the same set $V$ of $v$ vertices, valued by $W$. If $\mathcal{H}$ and $\mathcal{H}^{\prime}$ are $k$-hypomorphic up to $\mathfrak{G}$ then they are $t$ hypomorphic up to $\mathfrak{G}$.

\section{Conclusions}

The motivation of this line of research comes from several reconstruction results and conjectures about binary structures. The Ulam's reconstruction conjecture, still unsolved, is well-known (see the surveys [3, 4]). Fraïssé made a related conjecture about relational structures. The case of binary structures was handled by Lopez. A binary structure can be understood as a pair $R:=(V, c)$ where $c$ is a map from the set $V^{2}$ of ordered pairs $(x, y)$ of ele- 
ments of $V$ into a set $W$. The notion of isomorphism, and $k$-hypomorphism can be defined as for valued graphs. It was shown by Lopez [17] (see also [13]) that if two binary structures $R$ and $R^{\prime}$ on a finite set $V$ are $k$-hypomorphic for all $k \leq 6$ then they are $k$-hypomorphic for every integer $k$. Supposing $W=\{0,1\}$, Hagendorf and Lopez [15] say that two binary structures $R$ and $R^{\prime}$ are hemimorphic if either they are isomorphic or one is isomorphic to the dual of the other (the dual of $R:=(V, c)$ is $R^{d}:=\left(V, c^{d}\right)$ where $\left.c^{d}(x, y):=c(y, x)\right)$. They prove that hemimorphy behaves as hypomorphy, with a threshold of 12 instead of 6 . Numerous publications are built on these results (e.g. $[18,6,7,8]$ ). But for $W:=\{0,1\}^{n}$ and the corresponding notion of duality, the reconstruction problem is unsolved. Recently, Ben Amira, Chaari, Dammak and Si Kaddour [1] replace the dual of a binary structure $R:=(V, c)$ (with value in $W=\{0,1\}$ ) by its complement $R:=(V, \bar{c})$ defined by setting $\bar{c}(x, y):=1+c(x, y)$ (where the addition is defined modulo 2 ), they consider the notion of isomorphy up to complementation, and obtain some encouraging results. Instead of $W=\{0,1\}$ we may suppose that $W=\{0,1\}^{n}$, fix a group of permutations $\mathfrak{G}$ on $W$ and ask the same question as in Problem 3. As it is easy to see, results mentionned above are special instances of this question. On an other hand, none of these results extend to ternary relations [23]. Since the relation associated to a $h$-uniform hypergraph has arity $2 h$, the answer to Problem 3 does not seem to follow from general results.

\section{Acknowledgements}

The authors are pleased to thank the referees of this paper for their careful examination and helpful suggestions.

\section{References}

[1] A. Ben Amira, B. Chaari, J. Dammak and H. Si Kaddour, A reconstruction problem of digraphs under the hypomorphy up to complementation, work in progress, Lyon, December 2014.

[2] C. Berge and R. Rado, Note on isomorphic hypergraphs and some extensions of Whitney's theorem to families of sets, J. Combinatorial Theory Ser. B 13 (1972), 226-241. MR0311510

[3] J. A. Bondy, A graph reconstructor's manual. Surveys in combinatorics, 1991 (Guildford, 1991), 221-252, London Math. Soc. Lecture Note Ser., 166, Cambridge Univ. Press, Cambridge, 1991. MR1161466 
[4] J. A. Bondy and R. L. Hemminger, Graph reconstruction - a survey, J. Graph Theory 1 (1977), no. 3, 227-268. MR0480189

[5] J. A. Bondy and U. S. R. Murty, Graph theory. Graduate Texts in Mathematics, 244. Springer, New York, 2008, xii+651 pp. MR2368647

[6] Y. Boudabbous, La 5-reconstructibilité et l'indécomposabilité des relations binaires, European J. Combin. 23 (2002), no. 5, 507-522. MR1931935

[7] Y. Boudabbous and G. Lopez, The minimal non- $(\leq k)$-reconstructible relations, Discrete Math. 291 (2005), no. 1-3, 19-40. MR2124052

[8] B. Boushabi and A. Boussaïri, Les graphes (-2)-monohémimorphes, C. R. Math. Acad. Sci. Paris 350 (2012), no. 15-16, 731-735. MR2981342

[9] J. Dammak, G. Lopez, M. Pouzet and H. Si Kaddour, Hypomorphy of graphs up to complementation, J. Combin. Theory Ser. B 99 (2009), no. 1, 84-96. MR2467820

[10] J. Dammak, G. Lopez, M. Pouzet and H. Si Kaddour, Boolean sum of graphs and reconstruction up to complementation, Advances in Pure and Applied Mathematics 4 (2013), 315-349. MR3245475

[11] N. J. Fine, Binomial coefficients modulo a prime, Amer. Math. Monthly 54 (1947), no. 1, 589-592. MR0023257

[12] R. Fraïssé, Theory of relations. Revised edition. With an appendix by Norbert Sauer. Studies in Logic and the Foundations of Mathematics, 145. North-Holland Publishing Co., Amsterdam, 2000. ii+451 pp. (1st ed. 1986). MR1808172

[13] R. Fraïssé and G. Lopez, La reconstruction d'une relation dans l'hypothèse forte: Isomorphie des restrictions à chaque partie stricte de la base, SMS, Les presses de l'université de Montréal (1990). MR1060529

[14] D. H. Gottlieb, A class of incidence matrices, Proc. Amer. Math. Soc. 17 (1966), 1233-1237. MR0204305

[15] J. G. Hagendorf and G. Lopez, La demi-reconstructibilité des relations binaires d'au moins 13 éléments, C.R. Acad. Sci. Paris Sér. I Math. 317 (1993), no. 1, 7-12. MR1228954

[16] W. Kantor, On incidence matrices of finite projection and affine spaces, Math. Zeitschrift 124 (1972), 315-318. MR0377681 
[17] G. Lopez, Deux résultats concernant la détermination d'une relation par les types d'isomorphie de ses restrictions, C.R. Acad. Sci. Paris, Série A 274 (1972), 1525-1528. MR0369077

[18] G. Lopez and C. Rauzy, Reconstruction of binary relations from their restrictions of cardinality 2,3,4 and (n-1), II, Z. fur Math. Logik 38 (1992), 157-168. MR1254416

[19] E. Lucas, Sur les congruences des nombres eulériens et les coefficients différentiels des fonctions trigonométriques suivant un module premier, Bull. Soc. Math. France 6 (1878), 49-54. MR1503769

[20] D. Oudrar, Sur l'énumération de structures discrètes. Une approche par la théorie des relations; document de travail, Université d'Alger, Août 2014 .

[21] D. Oudrar and M. Pouzet, Décomposition monomorphe des structures relationnelles et profil de classes héréditaires, 7 pp. 2014, arXiv:1409.1432.

[22] M. Pouzet, Application d'une propriété combinatoire des parties d'un ensemble aux groupes et aux relations, Math. Zeitschr. 150 (1976), no. 2, 117-134. MR0460431

[23] M. Pouzet, Relations non reconstructibles par leurs restrictions, $J$. Combin. Theory Ser. B 26 (1979), no. 1, 22-34. MR0525814

[24] M. Pouzet and N. M. Thiéry, Some relational structures with polynomial growth and their associated algebras I: Quasi-polynomiality of the profile, Electron. J. Combin. 20 (2013), no. 2, Paper 1, 35 pp. MR3066340

[25] R. M. Wilson, A diagonal form for the incidence matrices of $t$-subsets vs. k-subsets, European J. Combin. 11 (1990), no. 6, 609-615. MR1078717

Maurice Pouzet

ICJ, MAThÉmATiques

Université Claude-Bernard LyOn1

43 Bd 11 Novembre 1918

69622 Villeurbanne Cedex

FRANCE

E-mail address: pouzet@univ-lyon1.fr

AND

Mathematics \& Statistics Department

UNIVERSITY OF CALGARY

Calgary, Alberta, T2N 1N4

CANADA 
HAMZA Si KADDOUR

ICJ, MathÉmatiques

Université Claude-Bernard Lyon1

43 BD 11 Novembre 1918

69622 Villeurbanne Cedex

FRANCE

E-mail address: sikaddour@univ-lyon1.fr

RECEIVED 14 JAnuARY 2015 